\title{
Noether's Conservation Laws and Stability in Nonlinear Conservative Interactions
}

\author{
Lisa Uechi ${ }^{1,2}$, Hiroshi Uechi ${ }^{3}$ \\ ${ }^{1}$ Institute of Industrial Science, The University of Tokyo, Tokyo, Japan \\ ${ }^{2}$ Present Address: Pacific Northwest Diabetes Research Institute, Seattle, USA, \\ ${ }^{3}$ Osaka Gakuin University, Osaka, Japan \\ Email: luechi@pnri.org, uechi@ogu.ac.jp
}

Received 31 March 2016; accepted 26 July 2016; published 29 July 2016

Copyright (C) 2016 by authors and OALib.

This work is licensed under the Creative Commons Attribution International License (CC BY). http://creativecommons.org/licenses/by/4.0/

(c) (†) Open Access

\begin{abstract}
We reviewed a nonlinear dynamical model in $\mathbf{2 n}$-variables which has conservative nonlinear interactions defined in terms of Noether's theorem in dynamics. The 2-variable $(n=1)$ conservative nonlinear model with external perturbations produced a possible explanation for problems such as the 10-year cycles of Canadian Lynx and snowshoe hare, interactions of microbes, stability and conservation law of nonlinear interacting systems. In this paper, the atto-fox $\left(1^{-18}\right.$-fox $)$ problem on the LV nonlinear equation, properties of 4-variable conservative nonlinear interactions different from nonconservative nonlinear interactions are examined and emphasized. Properties of the 4-variable $(n=2)$ conservative interaction model and a method to construct numerical solutions are discussed by employing the 2-variable solution. The periodic times of component variables and the net periodic time defined by superposition of component variables are discussed in order to study stability of the net 4-variable system. With symmetries and conservation laws, nonlinear analyses would be useful to study microscopic and macroscopic complex systems.
\end{abstract}

\section{Keywords}

Noether's Theorem, Conservative Nonlinear Model, Conservation Laws and Stability in Complex Systems

\section{Subject Areas: Biophysics}

\section{Introduction}

The concepts of stability, conservation law, symmetry are important in order to understand natural phenomena in physical, biological and engineering systems [1]-[6]. In our previous study, we proposed a conservative nonlinear dynamical model in $2 n$-variables which is primarily for nonlinear competitive interactions. The model provided a method for studying the relation between a conservation law and stability of a $2 n$-dimensional 
competitive system and restoration phenomena [7] [8].

Though symmetry and conservation laws have important information on nonlinear systems [9] [10], it is not possible to know at the outset what kind of conservation laws and nonlinearity should exist in complicated interacting systems, such as ecosystems of mammals [11] [12], microbes [13], systems of cells and organs [14] [15]. Nonlinear dynamical systems are characterized by self-interactions, dissipative structure, nonlinear cooperative phenomena. They are highly evolved and interacting systems.

Complex systems in natural sciences from microbiology to ecology, economy and environmental sciences are regarded as realizations of nonlinear dynamical interactions. Nonlinear phenomena are difficult to handle because of their complex interactions and structures, self-organizations, spontaneous emergence of order, which exhibit no simple laws or orders. However, complex systems constitute cells, organs, and living animals which make stable systems. There may be some conservation laws corresponding to the stability observed in microbiological systems. This is a motive that we applied nonlinear analyses based on Noether's theorem to dynamics, and interesting results for complex phenomena are discussed [7] [8].

Our approach to nonlinear phenomena based on conservation laws is briefly reviewed for 2-variable model $(n=1)$, and the solution of 2-variable nonlinear equation is used to construct a solution of the 4-variable $(n=2)$ conservative nonlinear equation. The approximate periodic amplitude and time of 4-variable nonlinear solutions are employed to explain stability of the net 4-variable system. The analysis of nonlinear systems with conservation laws would help understand complex systems on a fundamental level.

The conventional nonlinear approach is reviewed in Section 1.1, and the atto-fox problem of Lotka-Volterra (LV) type nonlinear equation is explained in Section 1.2. Then, a new approach with conservation law and stability is explained in Section 1.3. In Section 2, the time-dependent perturbations and restoration from the perturbation are discussed with applications to the 10-year cycle of Canadian lynx and snowshoe hare and food-web of microbes in Okanagan Lake. In Section 3, the conservative nonlinear 2-variable model is extended to the 4-variable conservative model and properties of the 4-variable calculations are discussed. Conclusion and remarks are in Section 4.

\subsection{Conventional Nonlinear Differential Equations for Interacting Systems}

It is often explained that a model of interacting populations is written in general as,

$$
\begin{aligned}
& \frac{\mathrm{d} x}{\mathrm{~d} t}=x f(x, y) \\
& \frac{\mathrm{d} y}{\mathrm{~d} t}=y g(x, y),
\end{aligned}
$$

where $f(x, y)$ and $g(x, y)$ are two autonomous differential equations which indicate that the time variable $t$ does not appear explicitly in the functions $f(x, y)$ and $g(x, y)$ with the condition $f(0,0)=0$ and $g(0,0)=0$. The meaning of functions depends on problems, where the nonlinear equations are applied, and this model is known as a general differential equation often called Kolmogorov's predator-prey model [16].

The simple and important applications in the field of ecology are those of Malthus (1959) for a population analysis, A. Lotka (1925) [17] and V. Volterra (1926) [18] for predator-prey differential equations known as Lotka-Volterra (LV) equation. The nonlinear equations have been applied to vast fields, such as biological pattern formations [19]-[21], restoration phenomena from injuries [22]-[24], epidemiology [25], branching processes of growth and extinction of populations [26], the 10-year cycle of Canadian lynx and snowshoe hare [27]-[33], the food chain of microbes in a lake [34] [35], economy and Lanchester strategic management [36] [37], and so forth.

The mathematical expressions of $f(x, y)$ and $g(x, y)$ in differential Equation (1) have been proposed and physical meanings of $f(x, y), g(x, y)$ and stability as an equilibrium are investigated in terms of limit cycles and atractors of Lyapunov functions [38] [39]. Lyapunov functions are scalar functions denoted as $V_{1}(t, x, y)$ and $V_{2}(t, x, y)$, instead of $f(x, y)$ and $g(x, y)$ in (1), and nonlinear functions are generalized to include $t$ with the condition $V_{1}(t, 0,0)=0$ and $V_{2}(t, 0,0)=0$. There are two types of Lyapunov functions which are strict Lyapunov (having negative definite time derivative along trajectories) and non-strict Lyapunov functions (negative semi-definite time derivatives along trajectories) [38]. The notion indicates how strongly a nonlinear system approaches to its equilibrium state when $t \rightarrow \infty$. In other words, a nonlinear interacting system in the 
Lyapunov type corresponds to a dissipative system or a nonconservative system leading to an equilibrium after a long time. This property is different from $2 n$-variable conservative nonlinear differential equations [7] [8].

\subsection{Note on the Atto-Fox (10-18-Fox) Problem on the Lotka-Volterra Equation}

The typical Lotka-Volterra (LV) equation used in applications is given by the following simple nonlinear equation:

$$
\begin{aligned}
& \frac{\mathrm{d} x}{\mathrm{~d} t}=x(\alpha-\beta y) \\
& \frac{\mathrm{d} y}{\mathrm{~d} t}=y(-\gamma+\delta x),
\end{aligned}
$$

where $x$ and $y$ are the population number of prey and predator respectively; $\mathrm{d} x / \mathrm{d} t$ and $\mathrm{d} y / \mathrm{d} t$ represent variations of populations with respect to time. The constants, $\alpha, \beta, \delta, \gamma$, are arbitrary nonlinear coefficients whose interpretations depend on problems at hand, and different nonlinear terms are readily included in the equation. It is not generally possible to obtain an analytical solution to the nonlinear differential equation, and a solution to (1.2) must be obtained by numerical computations with given coefficients as shown in the Figure 1 and Figure 2.

However, the nonlinear LV equation of the type (1.2) has an intrinsic problem known as atto-fox $\left(10^{-18}\right.$-fox) problem [40] [41] which is readily explained in the Figure 2. As we know in a common sense, living animals

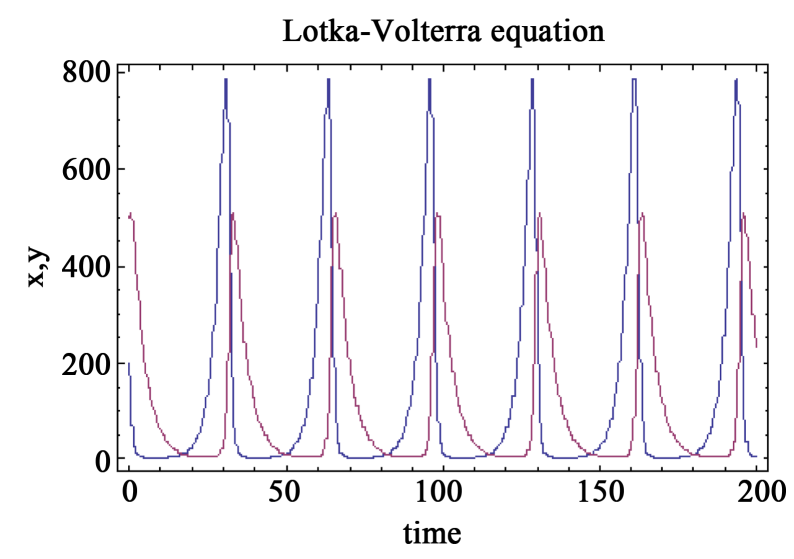

Figure 1. The values of nonlinear coefficients are, $\alpha=0.35$, $\beta=0.0035, \gamma=0.25, \delta=0.0020$ with initial values, $x(0)=200$ and $y(0)=500$.

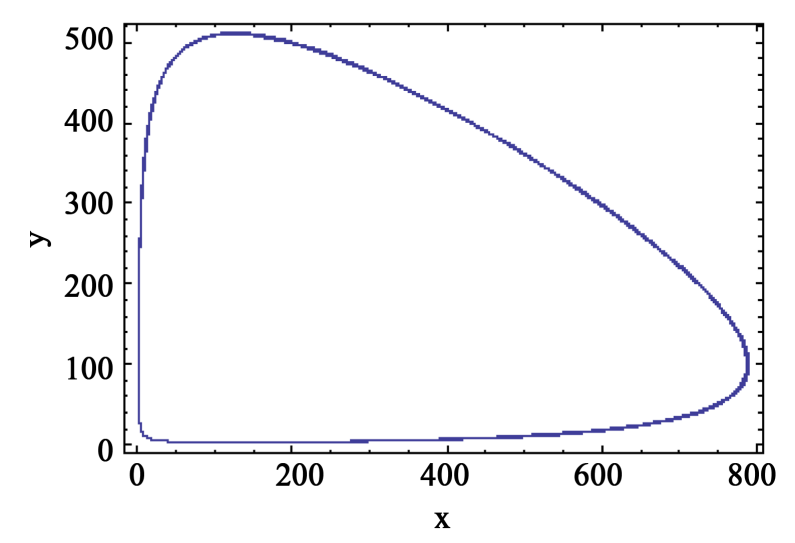

Figure 2. The $x-y$ phase space solution. 
interact with other animals through reasonably large integer numbers, however, the lower left of the Figure 2 shows that the small numbers such as $(x, y) \sim(1,90)$ at certain time $t$ can interact with each other and continue to live, which is absurd and meaningless.

More specifically, the LV equation (2) is invariant with the scale change:

$$
X(t)=a x(t), \quad Y(t)=b y(t),
$$

where $a$ and $b$ are arbitrary numbers. The equation for $X(t)$ and $Y(t)$ with $\beta^{\prime}=\beta / b$ and $\delta^{\prime}=\delta / a$ is given by:

$$
\begin{aligned}
& \frac{\mathrm{d} X}{\mathrm{~d} t}=X\left(\alpha-\beta^{\prime} Y\right) \\
& \frac{\mathrm{d} Y}{\mathrm{~d} t}=Y\left(-\gamma+\delta^{\prime} X\right) .
\end{aligned}
$$

Hence, the solution $(x(t), y(t))$ in Figure 2 can be equivalently constructed in the phase space $X(t), Y(t)$. Because of the arbitrariness of $a$ and $b$, if we choose, for example, $a=10^{18}$ and $b=1$, the solution can be expressed as $x(t)=10^{-18} X(t)$ and $y(t)=Y(t)$. The solution $(x(t), y(t))=\left(10^{-18} X(t), Y(t)\right)$ is obtained, and the atto-fox $\left(10^{-18}\right.$-fox) problem is proved. The LV equation of the type (1.2) has a serious potential problem when it is applied to real biological and ecological problems.

The problem of LV equations may be summarized as:

(a) the LV Equation (1.2) is too simple to apply to ecological and interactive systems.

(b) there are no restrictions on the nonlinear coefficients, $\alpha, \beta, \gamma, \delta$, whose values would be only determined by experimental data through numerical simulations. However, the arbitrariness of nonlinear coefficients produces a serious problem (the atto-fox problem).

(c) density-independent external perturbations should be included in numerical simulations in order to include changes of climate, temperature, seasons and landscape and so forth. The external perturbations are independent of $x(t)$ and $y(t)$.

Hence, it is necessary to find a new approach to resolve (a)-(c), which restricts values of nonlinear coefficients and numerically simulates real data with external perturbations.

\subsection{A New Approach with Conservation Laws and Stability}

It may seem natural to assume that a nonlinear system eventually dissipates energy (or an activation of the system) and arrives at an equilibrium state in the long run. However, the equilibrium state evolved as a dissipative process should be a maximum entropy state, and if the state needs to be activated, it needs some external inputs of energy. Hence, a nonlinear system demands certain external density-independent inputs or external perturbations which are not included in Lotka-Volterra and Kolmogorov type nonlinear Equations (1.1) and (1.2).

There are numerous examples of stable dynamical systems from microbiological systems such as DNA and RNA, amino acids and proteins, cells and organs, to macroscopic systems such as the 10-year cycle of Canadian lynx and snowshoe hare, wolves and caribous, creatures in marine coral reefs. The stability suggests that some conservation laws and symmetries are inherent from complex microbiological systems to macroscopic ecological systems. Hence, it could be possible to investigate conservation laws corresponding to a biological stability, which leads to a fundamental assumption for the model of conservative binary-coupled interactions [7] [8] based on Noether's theorem in dynamical systems [42]-[47].

The meaning of a conservation law in biological complex systems may be very different from conservation laws in physics described by way of a Lagrangian or a Hamiltonian. It is difficult to use direct mechanical concepts and analogies such as potential and kinetic energy, but it should be useful to employ the concept of conservation law and symmetry in terms of Noether's theorem in order to investigate conservation laws corresponding to biodiversity. Conservation laws and stability in biological systems could be employed to protect, understand and sustain the biosphere.

By employing the 2-variable conservative nonlinear model [7], it is possible to numerically simulate biological data such as the 10-year cycle of Canadian lynx and snowshoe hare, microbe food-web in the Okanagan lake [8]. We review briefly the 2-variable conservative nonlinear interactions, because solutions of 
the 2-variable model is used to construct the solution of the 4-variable conservative nonlinear model.

The Lagrangian of 2-variable conservative nonlinear system is described with the following Lagrangian,

$$
\mathcal{L}=\alpha_{1} \dot{x}_{1} x_{2}+\alpha_{2} x_{1} \dot{x}_{2}+\alpha_{3} x_{1}^{2}+\left(\alpha_{4}+\alpha_{5}\right) x_{1} x_{2}+\alpha_{6} x_{2}^{2}+\alpha_{7} x_{1}^{2} x_{2}+\alpha_{8} x_{1} x_{2}^{2} .
$$

From (1.5), we get the following nonlinear differential equation,

$$
\begin{aligned}
& \dot{x}_{1}=\frac{1}{d_{21}}\left\{\left(\alpha_{4}+\alpha_{5}\right) x_{1}+2 \alpha_{6} x_{2}+2 \alpha_{8} x_{1} x_{2}+\alpha_{7} x_{1}^{2}\right\}, \\
& \dot{x}_{2}=\frac{1}{d_{12}}\left\{2 \alpha_{3} x_{1}+\left(\alpha_{4}+\alpha_{5}\right) x_{2}+2 \alpha_{7} x_{1} x_{2}+\alpha_{8} x_{2}^{2}\right\} .
\end{aligned}
$$

The parameter $d_{21}$ is given by $d_{21}=\alpha_{2}-\alpha_{1}=-d_{12}$.

The conserved function, $\Psi$, of this system is given by,

$$
\Psi \equiv \alpha_{3} x_{1}^{2}+\left(\alpha_{4}+\alpha_{5}\right) x_{1} x_{2}+\alpha_{6} x_{2}^{2}+\alpha_{7} x_{1}^{2} x_{2}+\alpha_{8} x_{1} x_{2}^{2} .
$$

The Psi-function, $\Psi$, may correspond to the Hamiltonian or the Lagrangian in a mechanical system, however, one should note that the $\Psi$-function does not have any velocity (time-derivative) terms, showing no individual mechanical energy terms. The terms of the $\Psi$-function look like potential energy terms in order to conserve the $\Psi$-function as a constant value, whereas the interpretation of the function depends on nonlinear systems.

The solutions $x_{1}$ and $x_{2}$ are rapidly changing in time as shown in Figure 3 (ND1) and Figure 4 (ND2), which are computed as examples with parameters and initial starting values explained in each figure captions. One should be careful that solutions are sensitive to the value of chosen nonlinear parameters and initial starting values. The nonlinear parameters and initial values cannot be changed arbitrary and should be chosen carefully by numerical simulations with external perturbations.

The solutions in the phase space, $x_{1}$ and $x_{2}$, are shown in Figure 5, which are closed lines denoted as ND1 and ND2. The closed line in the phase space of solutions show that solutions respectively have definite periodic time. Though solutions $\left(x_{1}, x_{2}\right)$ are rapidly changing in time, the conserved function, $\Psi$, of nonlinear solutions is constant in time as shown in Figure 6. When the solution of conservative nonlinear equation cannot exist or be found with given parameters, the $\Psi$-function diverges or is simply not constant. This is helpful to examine the accuracy of numerical solutions to conservative nonlinear differential equations.

In widely used nonlinear differential equations, the eight interaction terms on the right-hand sides of (1.6) and (1.7) require eight independent parameters $\left(d_{12}\right.$ and $d_{21}$ are common factors and irrelevant). However, in case of the nonlinear interactions, regarding $\left(\alpha_{4}+\alpha_{5}\right)$ as one parameter, we have only five independent parameters at

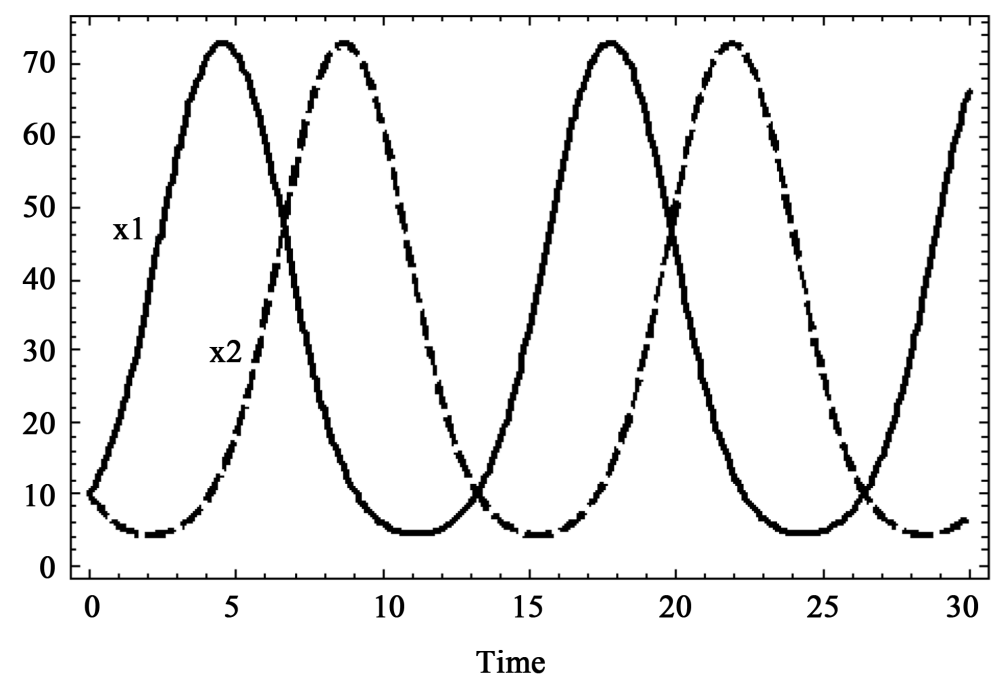

Figure 3. Numerical simulation of 2-variable ND1. The parameters of 2-variable ND1: $\alpha_{1}=1.0, \alpha_{2}=2.0, \alpha_{3}=-0.01, \alpha_{4}=0.5, \alpha_{5}=0.5, \alpha_{6}=-0.01$, $\alpha_{7}=-0.01, \quad \alpha_{8}=-0.01, \quad$ initial values are $x_{1}=-10.0, \quad x_{2}=-10.0$. 


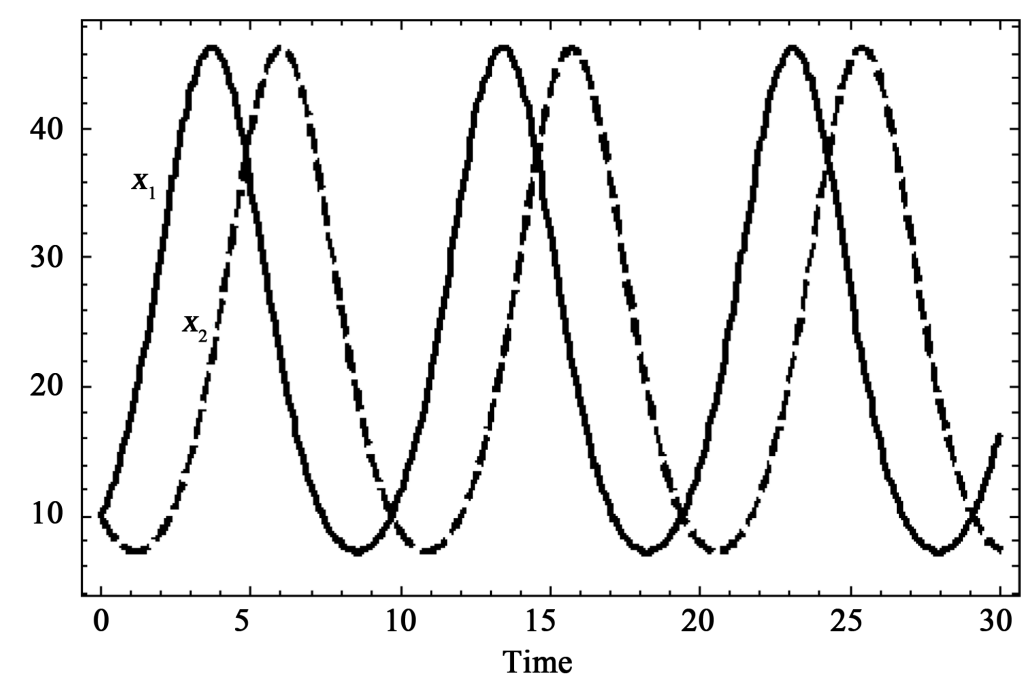

Figure 4. Numerical simulation of 2-variable ND1. The parameters of 2-variable ND2: $\alpha_{1}=1.0, \alpha_{2}=2.0, \quad \alpha_{3}=-0.1, \quad \alpha_{4}=0.5, \quad \alpha_{5}=0.5, \quad \alpha_{6}=-0.1$, $\alpha_{7}=-0.01, \alpha_{8}=-0.01$, initial values are $x_{1}=10.0, x_{2}=10.0$.

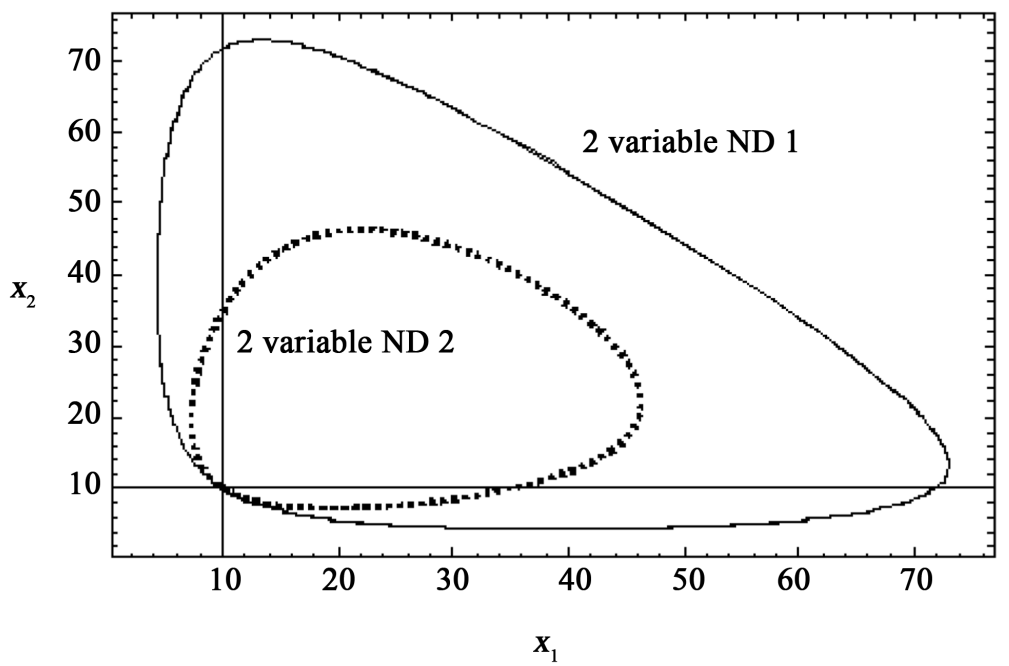

Figure 5. Numerical solutions of 2-variable ND1 and ND2.

the beginning in order to solve (1.6) and (1.7). This is helpful for numerical simulations of real data. The property of the $2 n$-variable conservative nonlinear equation is essentially different from properties of LotkaVolterra and Kolmogorov equations.

We would like to answer some questions concerning difficulties on nonlinear differential equations in order to elucidate applicability of the $2 n$-variable conservative nonlinear differential equations.

(Question 1) There may be infinitely many periodic solutions in the nonlinear model that depend on the values of nonlinear coefficients, $\alpha_{1}, \cdots, \alpha_{8}$, and initial starting values, $x_{1}(0), x_{2}(0)$.

(Answer) This is restated as that solutions should be transformed in any form of solutions in the phase space, $x_{1}, x_{2}$, resulting in the atto-fox problem or a physically meaningless problem. It may be correct for simple LV type nonlinear equations. However, one should be careful with the 2-variable conservative interaction model. The nonlinear coefficients and initial values in the 2-variable model are restricted as (1.6) and (1.7) by conservation law $\Psi=$ constant, and positivity $x_{i} \geq 0$ can be added as a constraint if necessary. Nonlinear coefficients and initial starting values are restricted in certain values and not entirely free adjustable parameters. The phase space solutions in Figure 5 of the 2-variable nonlinear model cannot be transformed continuously to 


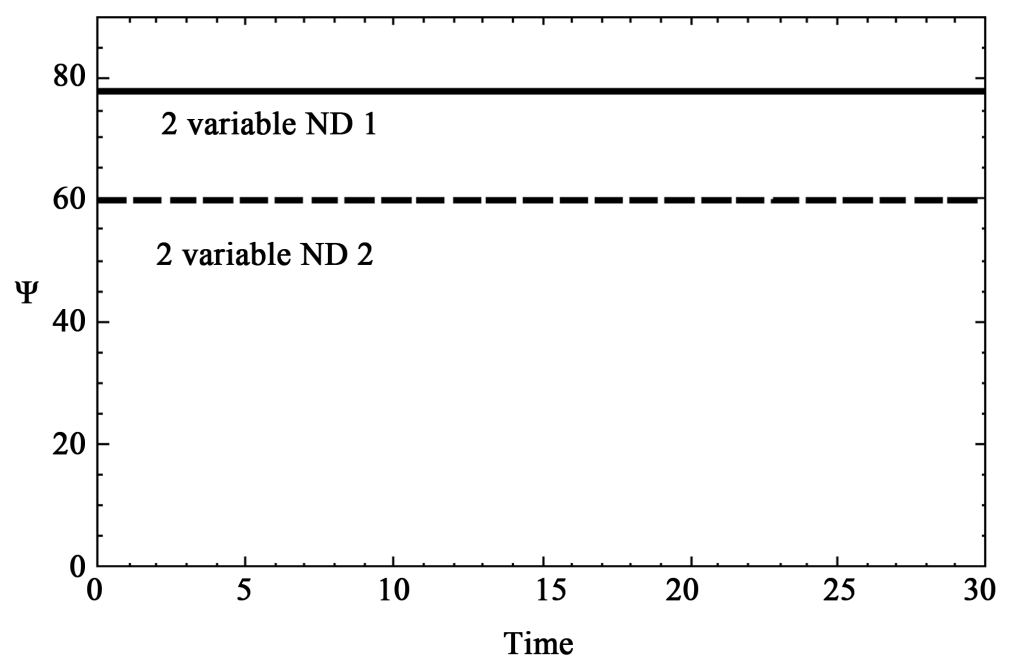

Figure 6. Numerical solutions of conserved quantity $\Psi$ of 2-variable ND1 and ND2. Note that $\Psi$ is constant in both cases.

those of the atto-fox $\left(10^{-18}\right.$-fox $)$ solutions.

When initial starting values and nonlinear coefficients are inconsistent, one obtains no solutions and the $\Psi$-function diverges [8]. At present, we do not know of any theoretical relations among values of nonlinear coefficients and initial starting values in conservative nonlinear equations. The consistent values of initial starting values and nonlinear coefficients should be determined from numerical simulations by including external perturbations. The $\Psi$-function can be used to check accuracy of numerical solutions.

(Question 2) Selecting initial conditions of scarce prey and plentiful predators, unphysical solutions to nonlinear equations could occur. For example, $x_{1}$ may decrease even if it is zero when $x_{2}$ is positive and increasing, and $x_{2}$ may decrease or increase even if $x_{1}$ is negative. This means that non-existing prey can be eaten by predators, and predators can increase or decrease by eating or interacting with negative prey.

(Answer) This is a typical surmise derived from LV type nonlinear equations, but the assumption is not correct at all with the conservative nonlinear model. Because nonlinear coefficients are not entirely free parameters, it is not possible to find solutions by selecting any nonlinear coefficients and initial conditions of scarce prey and plentiful predators, and atto-fox type problems are restricted. A specific numerical example is shown in the paper [8]. The solutions to $2 n$-variable conservative nonlinear equations are different from those of conventional, nonconservative nonlinear equations. The (Question 2) is correct if one is solving simple nonlinear equations with no constraints on nonlinear coefficients.

\section{Time-Dependent Perturbations, Restoration from Perturbations}

Lotka-Volterra and Kolmogorov equations are not sufficient to study nonlinear systems because they do not include density-independent external perturbations, such as climate and seasonal changes, pesticides and herbicides, hunting, forest fires and storms, etc. These external factors are density-independent and they are not expressible as (1.1). Hence, it is necessary to simulate density-independent and time-dependent external factors, and they are introduced as piecewise continuous, constant perturbations in the nonlinear equations discussed in detail in the papers [7] [8].

\subsection{Piecewise Continuous External Perturbations}

In order to investigate numerical responses of a system to external perturbations, piecewise continuous constants are introduced by using $\theta$-function such that

$$
c_{i}=f_{i}\left\{\theta\left(t-t_{\text {start }}\right)-\theta\left(t-t_{\text {end }}\right)\right\}, \quad(i=1,2),
$$

where $\theta\left(t-t^{\prime}\right)$ represents a step function: 


$$
\theta\left(t-t^{\prime}\right)= \begin{cases}1, & \left(t \geq t^{\prime}\right), \\ 0, & \left(t<t^{\prime}\right),\end{cases}
$$

and coefficients $f_{i}(i=1,2)$ are positive or negative constants to express strength of external perturbations.

An execution of an external perturbation to $\left(x_{1}, x_{2}\right)$, is shown in Figure 7 and Figure 8. The phase space solution denoted as St 1 (state 1) jumps to the solution Sp 1 (starting-point 1)-Ep 1 (end-point 1) when the external perturbation is switched on at the moment Sp 1, and goes back to the solution St 2 when the perturbation is switched off at Ep 1.

The instant that an external perturbation is added to conservative nonlinear equations, the solution jumps to possible another conserved system. When the perturbation was switched off (see, Ep 1 in Figure 8), the system recovered the original state or a possible stable state.

We gradually changed the strength and duration time of a negative external perturbation. The system advanced to unstable states and disintegrated, $x_{1}(t), x_{2}(t) \rightarrow-\infty$, corresponding to the extinction of species. The system cannot find solutions, $x_{1}(t), x_{2}(t)>0$, which is shown by a non-constancy of $\Psi$. Hence, there is a threshold for the negative external perturbation to each component, which makes the component amplitude negative and diverge, resulting in extinction of species [8].

The strength and duration time of negative external perturbations which make the system unstable were evaluated numerically. The threshold of negative perturbation is related to the amplitude and periodic time of each component, $x_{1}(t), x_{2}(t)$, and if the strength and duration time of an external perturbation are not large compared to the amplitude and periodic time of species, the nonlinear interacting system converges to a possible stable solution. It should be possible to recover a complex interacting system with the assistance of external perturbations if we know the standard rhythm [7] [8] or approximate amplitude and periodic time of component variables.

The threshold of strength and duration time of negative perturbations is important information to regulate nonlinear complex systems. The 2-variable conservative nonlinear model indicates that if we know the relation

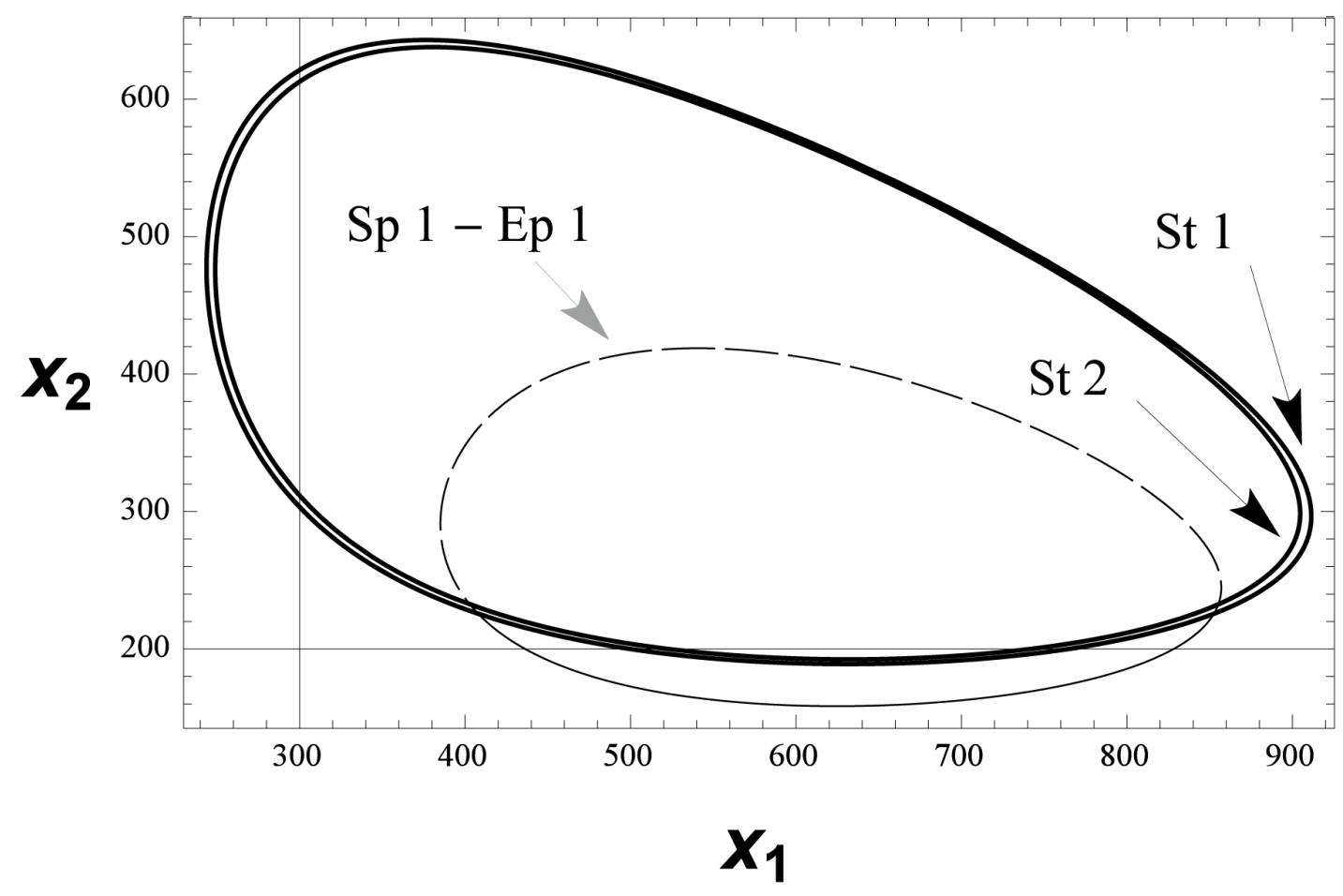

Figure 7. The $\left(x_{1}, x_{2}\right)$ phase-space transition with the negative perturbation as in (a). Solid line, St 1, (State 1) is the initial state, and St 2 (State 2) is the recovered state after the end of perturbation. Dashed line is phase-space during Sp 1 (starting-point 1)-Ep 1 (endpoint 1). 


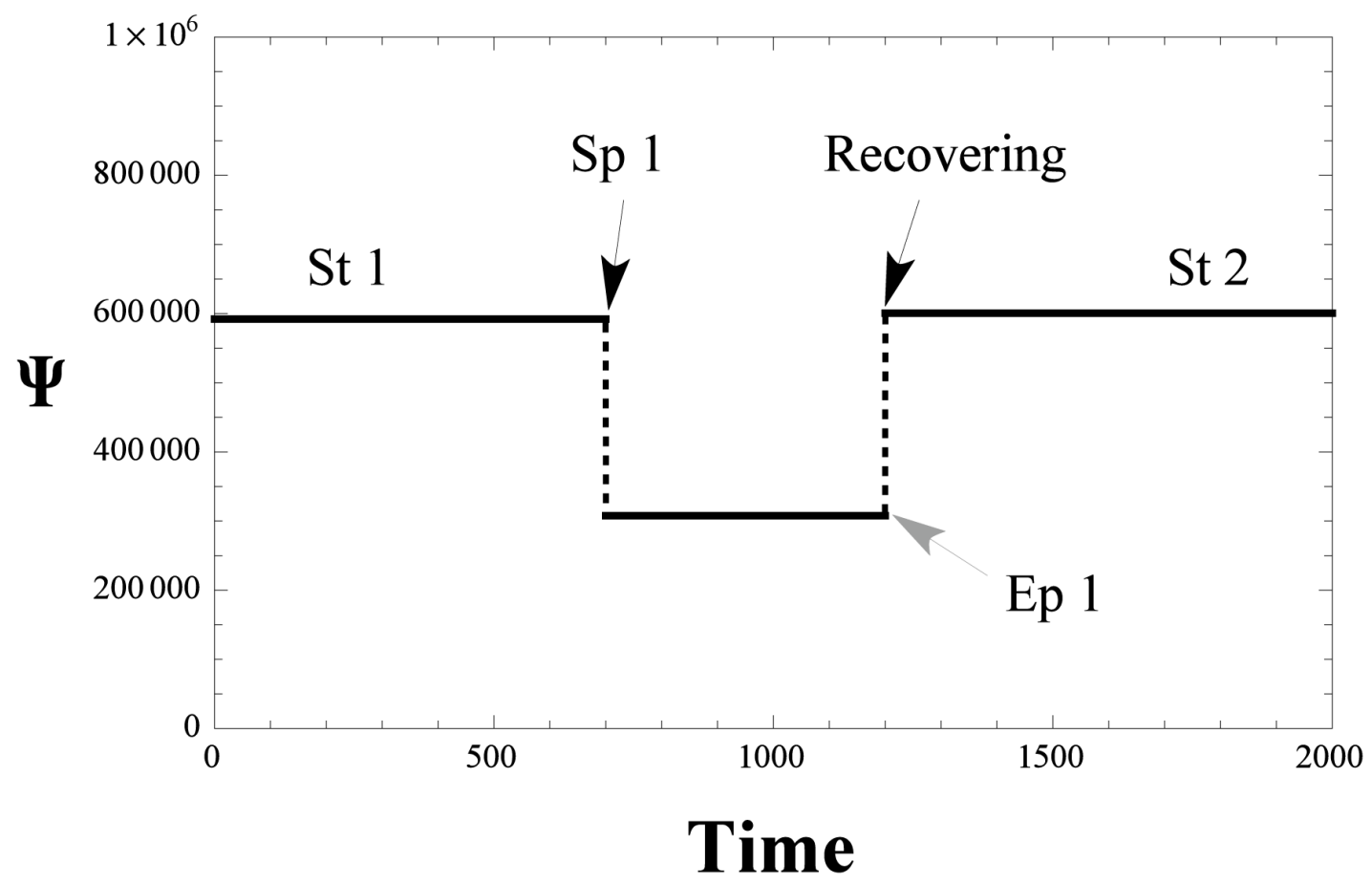

Figure 8. Conservation law $\Psi$ of 2 -variable ND with one perturbation. $\Psi$ changed $\Psi \simeq 60000$ to $\Psi \simeq 30000$ by introducing perturbation. It recovers after Ep 1 (end-point 1).

between stability and rhythm of interacting systems and exert appropriate external perturbations at a correct time, it could be possible to save species from extinction.

\subsection{The 10-Year Population Cycle of Canadian Lynx and Snowshoe Hare}

As a specific example, the 2-variable nonlinear model with perturbations is applied to analyses of the 10-year cycles of Canadian Lynx and snowshoe hare [27]-[33]. The 2-variable conservative nonlinear model with external perturbations can successfully simulate the reported data of the Lynx-hare, and we found that there is a stable cycle, the standard rhythm, inferred from the analysis of conservative nonlinear interactions. It is possible to understand the stability and standard rhythm of the 10-year cycles of Canadian Lynx and snowshoe hare in terms of a conservation law of a prey-predator system [8].

The standard rhythm is discussed in the paper [8], which explains stable population cycles, such as the 10year cycle of Canadian lynx and snowshoe hare and microbes' food-web in Okanagan Lake. The population cycles simulated by the current conservative nonlinear interaction are stable and show recovery and restoration from external perturbations as long as the environmental systems for living and interacting animals are not seriously damaged. Figure 7 is a numerical example of recovery and restoration phenomena.

The current conservative nonlinear model is applied to the data of food-web in Okanagan Lake [34] [35]; mysis introduction to the Lake is known as an effective method to enhance ecological interactions among microbes and other creatures so as to increase fisheries' production. The maximum and minimum densityamplitude of microbes are also well simulated with the 2-variable conservative nonlinear model, and the results suggest that stability and periodic restoration phenomena in ecosystems may be a manifestation of a conservation law with external perturbations.

The important property of the 2-variable nonlinear model with conservation law is that coupled systems can have certain recovering strength to external perturbations. As a predator needs a prey for its food, a prey needs a predator for the control and conservation of their own species. The conservation law and the standard rhythm of species are considered to be naturally constructed by species to live for a long time in nature. Therefore, the rhythm and stability of population cycle as a whole in an ecosystem would be interpreted as a manifestation of 
the survival of the fittest adapted to the balance in an ecosystem.

The stability and conservation law are constructed by species in mutual dependency or cooperation to survive for long-time periods in severe environmental conditions. The standard rhythm should be regarded as the result of strategy for species to live in nature. Whatever roles they have to play, the species that can fit and balance with other creatures can survive in nature. A strong predator cannot even survive if it ignores the law of the standard rhythm and conservation law of nature constructed by other members and the environment. The conservative BCF nonlinear model with perturbations enables one to examine a fundamental law of nature in a form of differential equations. We hope that this study will help understand both activities of animals and humans in natural life.

\section{The 4-Variable Dynamical Model}

Based on the reviews and discussions, we extend the 2-variable conservative model to the 4 -variable model for applications to more realistic interacting systems. Several examples can be found in microbiology and medical fields [14] [15]. Statistical methods are normally used to investigate correlations among physical data (morphogens, responses of cells, concentrations, populations, etc.). Dynamical analysis can be possible by applying the 4-variable conservative nonlinear differential equation.

A morphogen is considered a substance governing the pattern of tissue development in the process of morphogenesis, or a signaling molecule that acts directly on cells to produce specific cellular responses depending on its concentration. When morphogens are identified, a dynamical method based on the 2-variable or 4-variable conservative nonlinear dynamical analyses could be possible in microbiology.

However, the 4-variable nonlinear differential equations have too many nonlinear parameters to be set at the beginning, and they are difficult to solve and apply to numerical simulations. Therefore, we discussed a strategy to construct solutions to 4 -variable, 6 -variable, ..., conservative nonlinear differential equations. The construction of $2 n$-variable solution is based on the solution of 2 -variable solutions.

The 4-variable conservative nonlinear model for complex systems is described as:

$$
\begin{aligned}
\dot{x}_{1}= & \frac{1}{d_{21}}\left\{\left(\alpha_{6}+\alpha_{13}\right) x_{1}+2 \alpha_{14} x_{2}+\left(\alpha_{10}+\alpha_{15}\right) x_{3}+\left(\alpha_{16}+\alpha_{18}\right) x_{4}\right. \\
& \left.+\alpha_{21} x_{1}^{2}+2 \alpha_{22} x_{1} x_{2}+\alpha_{23} x_{1} x_{3}+\alpha_{24} x_{1} x_{4}+\alpha_{26} x_{3} x_{4}\right\}+\frac{c_{1}}{d_{21}}, \\
\dot{x}_{2}= & \frac{1}{d_{12}}\left\{2 \alpha_{5} x_{1}+\left(\alpha_{6}+\alpha_{13}\right) x_{2}+\left(\alpha_{7}+\alpha_{9}\right) x_{3}+\left(\alpha_{8}+\alpha_{17}\right) x_{4}\right. \\
& \left.+2 \alpha_{21} x_{1} x_{2}+\alpha_{22} x_{2}^{2}+\alpha_{23} x_{2} x_{3}+\alpha_{24} x_{2} x_{4}+\alpha_{25} x_{3} x_{4}\right\}+\frac{c_{2}}{d_{12}}, \\
\dot{x}_{3}= & \frac{1}{d_{43}}\left\{\left(\alpha_{8}+\alpha_{17}\right) x_{1}+\left(\alpha_{16}+\alpha_{18}\right) x_{2}+\left(\alpha_{12}+\alpha_{19}\right) x_{3}+2 \alpha_{20} x_{4}\right. \\
& \left.+\alpha_{24} x_{1} x_{2}+\alpha_{25} x_{1} x_{3}+\alpha_{26} x_{2} x_{3}+\alpha_{27} x_{3}^{2}+2 \alpha_{28} x_{3} x_{4}\right\}+\frac{c_{3}}{d_{43}}, \\
\dot{x}_{4}= & \frac{1}{d_{34}}\left\{\left(\alpha_{7}+\alpha_{9}\right) x_{1}+\left(\alpha_{10}+\alpha_{15}\right) x_{2}+2 \alpha_{11} x_{3}+\left(\alpha_{12}+\alpha_{19}\right) x_{4}\right. \\
& \left.+\alpha_{23} x_{1} x_{2}+\alpha_{25} x_{1} x_{4}+\alpha_{26} x_{2} x_{4}+2 \alpha_{27} x_{3} x_{4}+\alpha_{28} x_{4}^{2}\right\}+\frac{c_{4}}{d_{34}},
\end{aligned}
$$

where coupling constants, $\alpha_{1}, \alpha_{2}, \cdots, \alpha_{28}$, and $d_{12}=\alpha_{1}-\alpha_{2}, d_{21}=\alpha_{2}-\alpha_{1}=-d_{12}$, and $d_{34}=\alpha_{3}-\alpha_{4}$, $d_{43}=\alpha_{4}-\alpha_{3}=-d_{34}$ represent interaction strength among species $\left(x_{1}, x_{2}, x_{3}, x_{4}\right)$. The numbering of nonlinear coefficients, $\alpha_{i}$, is chosen for our convenience by starting from a lagrangian to generate (3.1)-(3.4). It is explained in the Appendix.

The external perturbations are expressed as arbitrary, piecewise continuous constants, $c_{1}, c_{2}, c_{3}, c_{4}$, which represent strengths of external effects (such as, temperature changes, reductions of species by huntings or 
fishings, etc., as cases may be). They are pulse-like constants expressed by step-functions.

The 4-variable conserved nonlinear equation is derived from Euler-Lagrange equation in Lagrangian dynamics. The Noether's theorem in dynamics is employed to obtain the conservation law in complicated nonlinear systems. Hence, coefficients of the right-hand side of (3.1)-(3.4) are restricted such that the system has conserved quantities.

In conventional 4-variable nonlinear differential equations of the type (3.1)-(3.4), nine terms in the right-hand side are supposed to be independent and adjusted freely. Hence, the number of nonlinear coefficients basically adds up to 40 parameters $\left(9 \times 4=36\right.$ and 4 parameters, $\left.c_{1}, c_{2}, c_{3}, c_{4}\right)$. In the current nonlinear equation, coefficients of the type, $\left(\alpha_{6}+\alpha_{13}\right), \cdots$, can be handled as one parameter, and the number of independent parameters adds up to 22 . There are still many free parameters, however, admissible solutions would be confined further when initial starting values and positivity, $x_{1}(t)>0, x_{2}(t)>0, x_{3}(t)>0, x_{4}(t)>0$, are included. These conditions and the conservation law, $\Psi=$ const. , are helpful for numerical simulations.

In order to make the 4-variable nonlinear equation useful for numerical simulations, we propose a strategy to construct a 4-variable solution by employing 2-variable solutions.

1) One should construct two sets of 2-variable numerical solutions independently by dividing interactions of 4 variables $\left(x_{1}, x_{2}, x_{3}, x_{4}\right)$ as 2-variables $\left(x_{1}, x_{2}\right)+2$-variables $\left(x_{3}, x_{4}\right)$. The two sets of the 2-variable conservative nonlinear solutions, $\left(x_{1}, x_{2}\right)$ and $\left(x_{3}, x_{4}\right)$, should be prepared from respective data. External perturbations could be included to create reasonable numerical simulations in the 2-variable form [7] [8].

2) The 2-variable numerical simulations, $\left(x_{1}, x_{2}\right)$, are regarded as solutions for (3.1) and (3.2) by assuming coefficients related to $x_{3}$ and $x_{4}$ as zero. Similarly, the 2-variable solutions, $\left(x_{3}, x_{4}\right)$, are also regarded as solutions for (3.3) and (3.4) by assuming coefficients related to $x_{1}$ and $x_{2}$ as zero. Hence, the value of coefficients in the 4-variable solution (3.1)-(3.4) are almost fixed at the beginning.

3) The next step is to weakly couple the set of 2-variable solutions by using the 4-variable solution (3.1)-(3.4). The weak coupling of $\left(x_{1}, x_{2}\right)$ and $\left(x_{3}, x_{4}\right)$ can be performed by changing cross-coupling coefficients (assumed zero) slowly from 0 to $0 \pm \epsilon \quad(\epsilon \sim 0.001)$. We have four coupling coefficients, $\alpha_{23}, \alpha_{24}, \alpha_{25}, \alpha_{26}$, and four coefficients from linear terms, $\left(\alpha_{10}+\alpha_{15}\right),\left(\alpha_{16}+\alpha_{18}\right),\left(\alpha_{7}+\alpha_{9}\right),\left(\alpha_{8}+\alpha_{17}\right)$, which can be regarded as four parameters. Hence, in the 4-variable conservative nonlinear equation, we are supposed to change eight parameters to numerically simulate data in question. By referring to combinations of data: $\left(x_{1}, x_{3}\right),\left(x_{1}, x_{4}\right),\left(x_{2}, x_{3}\right)$ and $\left(x_{2}, x_{4}\right)$, reasonable eight parameters should be searched in numerical simulations by trial and error.

The coupling constants, $\alpha_{23}, \alpha_{24}, \alpha_{25}, \alpha_{26}$, and four coefficients of linear terms characteristically change amplitude and wave-shape. Even if coupling constants are small, one would encounter divergences when nonlinear parameters and initial conditions are not compatible, which can be specifically perceived by checking the value of $\Psi$ function. The $\Psi$-function is very useful to check the existence of solutions.

Following theoretical discussions of invariant variational method in dynamics, the $\Psi$-function for the 4variable conserved nonlinear dynamical system is expressed as,

$$
\begin{aligned}
\Psi= & \alpha_{5} x_{1}^{2}+\left(\alpha_{6}+\alpha_{13}\right) x_{1} x_{2}+\left(\alpha_{7}+\alpha_{9}\right) x_{1} x_{3}+\left(\alpha_{8}+\alpha_{17}\right) x_{1} x_{4}+\left(\alpha_{10}+\alpha_{15}\right) x_{2} x_{3} \\
& +\alpha_{11} x_{3}^{2}+\left(\alpha_{12}+\alpha_{19}\right) x_{3} x_{4}+\alpha_{14} x_{2}^{2}+\left(\alpha_{16}+\alpha_{18}\right) x_{2} x_{4}+\alpha_{20} x_{4}^{2}+\alpha_{21} x_{1}^{2} \\
& +\alpha_{22} x_{1} x_{2}^{2}+\alpha_{23} x_{1} x_{2} x_{3}+\alpha_{24} x_{1} x_{2} x_{4}+\alpha_{25} x_{1} x_{3} x_{4}+\alpha_{26} x_{2} x_{3} x_{4} \\
& +\alpha_{27} x_{3}^{2} x_{4}+\alpha_{28} x_{3} x_{4}^{2}+c_{1} x_{2}+c_{2} x_{1}+c_{3} x_{4}+c_{4} x_{3} .
\end{aligned}
$$

The physical quantities $\left(x_{1}, x_{2}, x_{3}, x_{4}\right)$ change in time, but the sum of the combination given by (3.5) is constant in time. The strategy to find a solution, (1)-(3), and experiences with nonlinear differential equations help obtain a numerical solution to the system of nonlinear differential equations. A set of solution to (3.1)-(3.4) and the value of $\Psi$-function are shown in Figures 9(a)-(d).

The 4-variables $\left(x_{1}, x_{2}, x_{3}, x_{4}\right)$ are time-changing as in Figure 9(a), but $\Psi$-function is constant in time as shown in Figure 9(b), which confirms numerical solutions are accurate. The phase spaces of solutions for $\left(x_{1}(t), x_{2}(t)\right)$ and $\left(x_{3}(t), x_{4}(t)\right)$ are shown in Figure 9(c) and Figure 9(d), respectively. One can see that the phase space of solutions is confined in each volume of phase space. The other phase spaces of solutions are also shown in Figures 10(a)-(d).

\section{The Results of the 4-Variable Conservative Nonlinear Model}

The 2-variable nonlinear solution $\left(x_{1}(t), x_{2}(t)\right)$ shows a recurrence time, $T$, defined by $x(t+T)=x(t)$, and 
the simple closed line as Figure 5 indicates that the recurrence time of solution (periodicity) is finite. However, trajectories of solutions in the 4-variable conservative nonlinear model in Figure 9(c), Figure 9(d) and Figures 10(a)-(d) are not closed lines, but they enclose a finite area in each phase space. The solutions, $x_{1}, x_{2}, x_{3}, x_{4}$, oscillate within a finite, closed surface of phase space and exhibit approximate periodic time, $x_{i}\left(t+T_{i}\right) \sim x_{i}(t)$ $(i=1,2,3,4)$ produced by nonlinear interactions.

It is well known in linear differential equations that the net response (amplitude) $y$ at a given place and time is the sum of independent responses $\left(x_{1}, x_{2}, \cdots, x_{k}\right): y=\sum_{i=1}^{k} x_{i}$, which is known as the superposition principle. The superposition principle does not hold in nonlinear systems. However, we examined the net amplitude defined by $y=\sum_{i=1}^{4} x_{i}=x_{1}+x_{2}+x_{3}+x_{4}$, because the net amplitude may have important information for the total system.

In our conservative nonlinear interactions, we found that component solutions approximately produced a periodic shape of the net amplitude. The shape of the net amplitude is very complicated but oscillates at an approximate pitch like a resonance produced by a tuning fork on resonance box.

The approximate periodic times of each variable $x_{1}, x_{2}, x_{3}, x_{4}$ in Figure 9(a) are respectively within $400 \lesssim T_{i} \lesssim 500 \quad(i=1,2,3,4)$. The net periodic time $T_{n}$ is found from the resulting periodic shape of the amplitude, $y$, shown in Figure 11. The approximate net periodic time of the system is $T_{n} \sim 1000$ in the current example as observed in Figure 11. One can examine that the almost similar shape of wave pattern within $T_{n} \sim 1000$ is repeated, which is also checked by changing time-span longer in the numerical calculations.

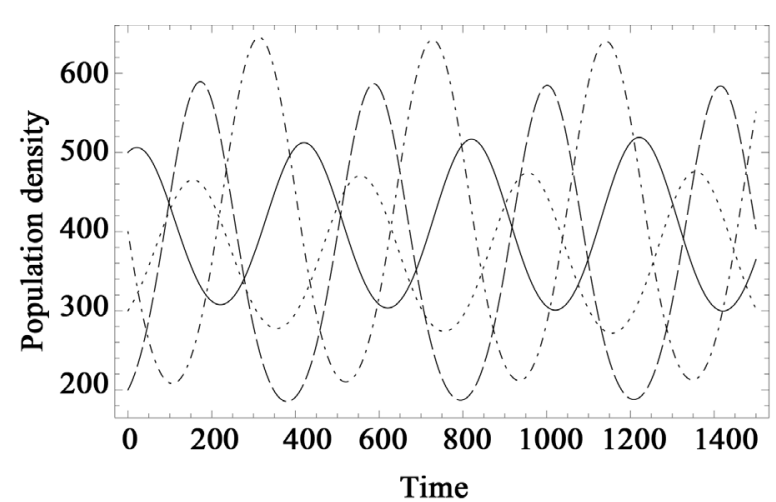

(a) 4-variable ND solution

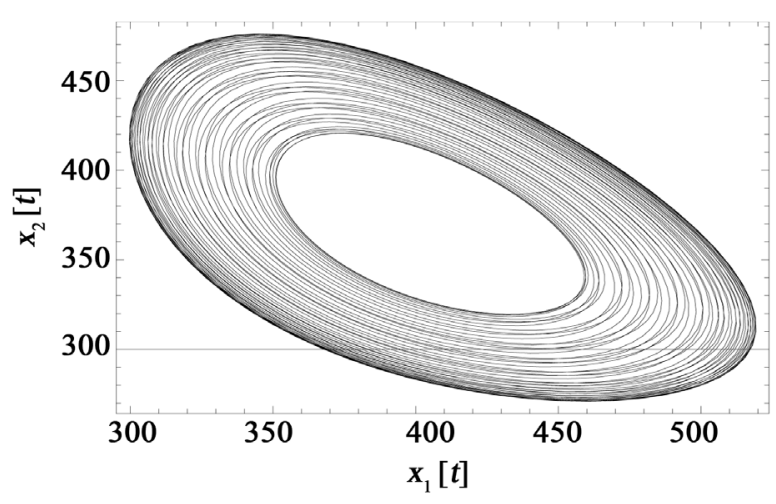

(c) Conservation law model of 4 -variable ND

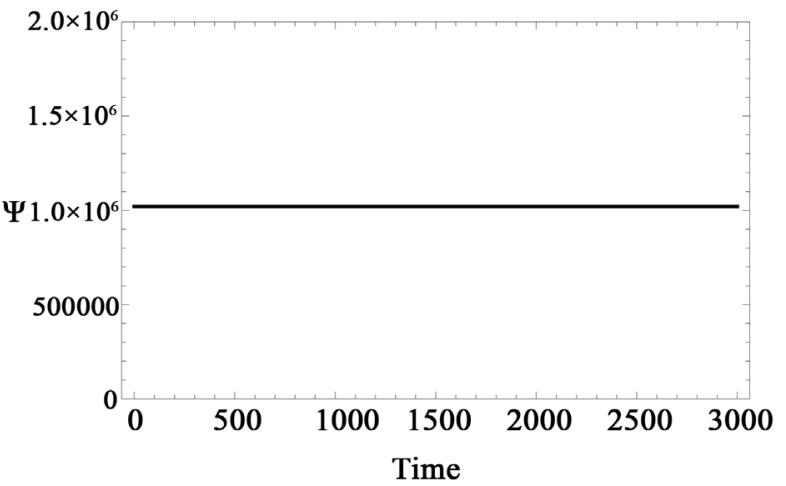

(b) Conservation law model of 4 -variable ND

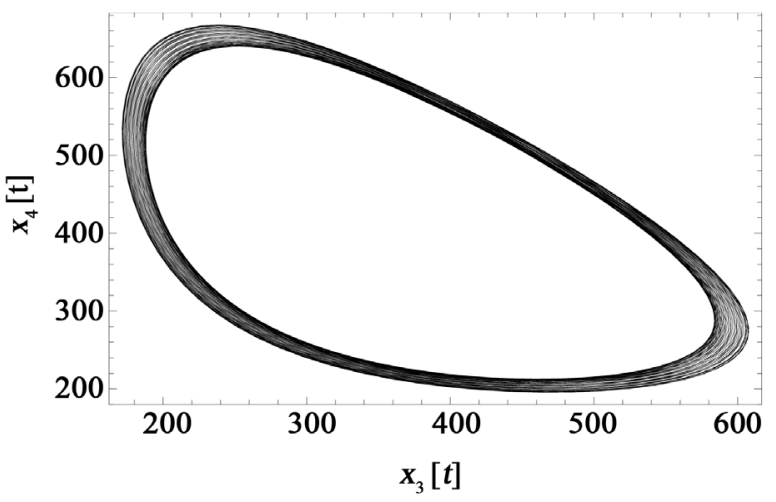

(d) Conservation law model of 4-variable ND

Figure 9. The solutions of 4-ND system and phase-space with respect to time. Figure 9(a) represents solutions and Figure 9(b) is the conservation law $\Psi$ with respect to time. Figure 9 (c) and Figure $9(d)$ are phase-space of solutions of $x_{1}$ and $x_{2}, x_{1}$ and $x_{4}$, respectively. 


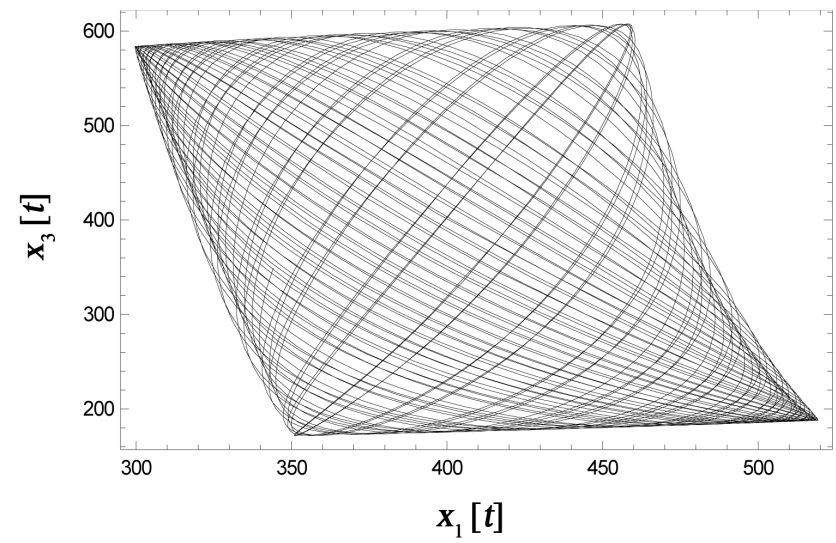

(a) Conservation law model of 4-variable ND

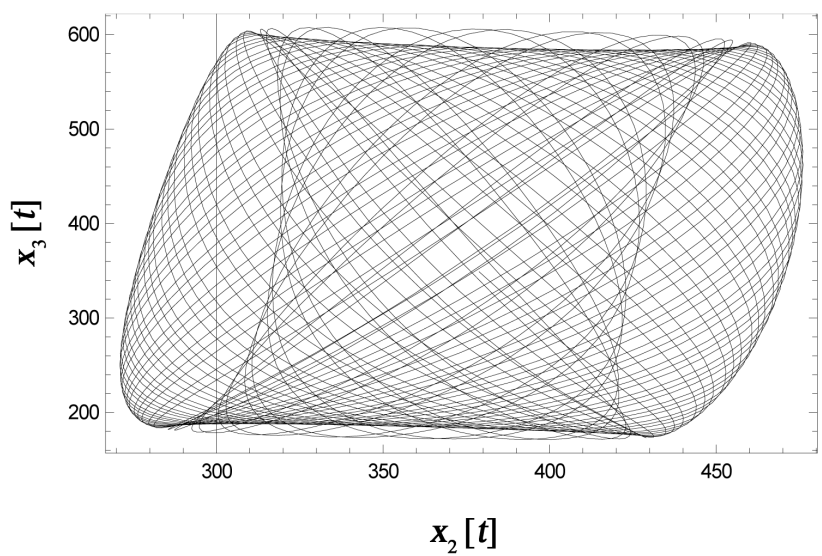

(c) Conservation law model of 4-variable ND

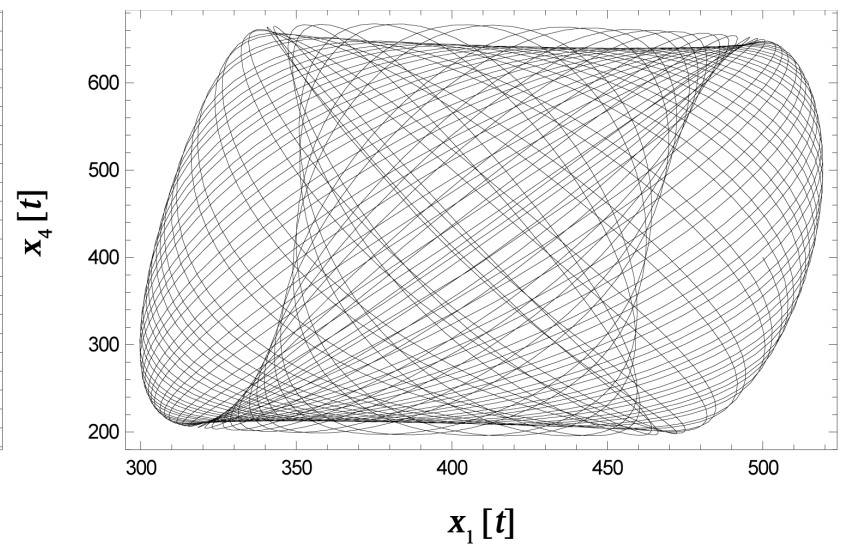

(b) Conservation law model of 4-variable ND

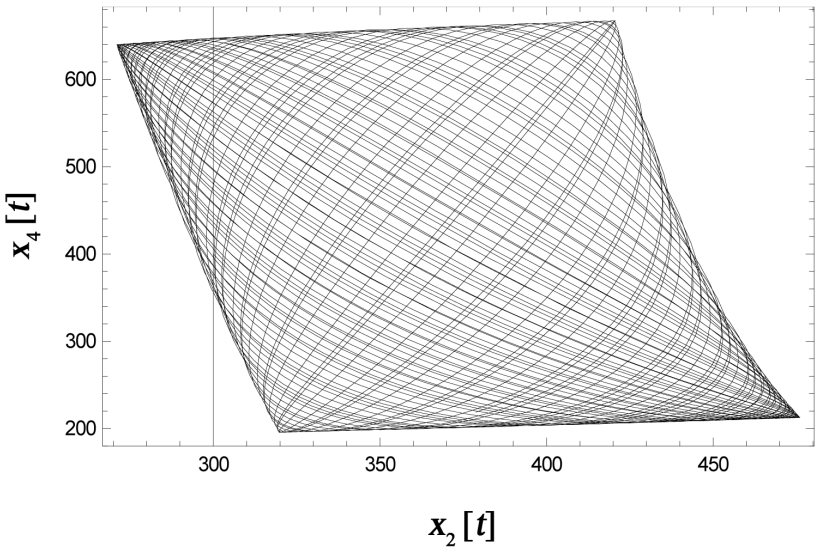

(d) Conservation law model of 4-variable ND

Figure 10. The phase-space of 4-variable ND model for another combination of solutions. Figure 10(a) represents phase-space of $x_{1}$ and $x_{3}$, Figure 10(b) is a phase space of $x_{1}$ and $x_{4}$. Figure 10(c) and Figure 10(d) are phase-space of solutions of $x_{2}$ and $x_{3}, x_{2}$ and $x_{4}$, respectively.

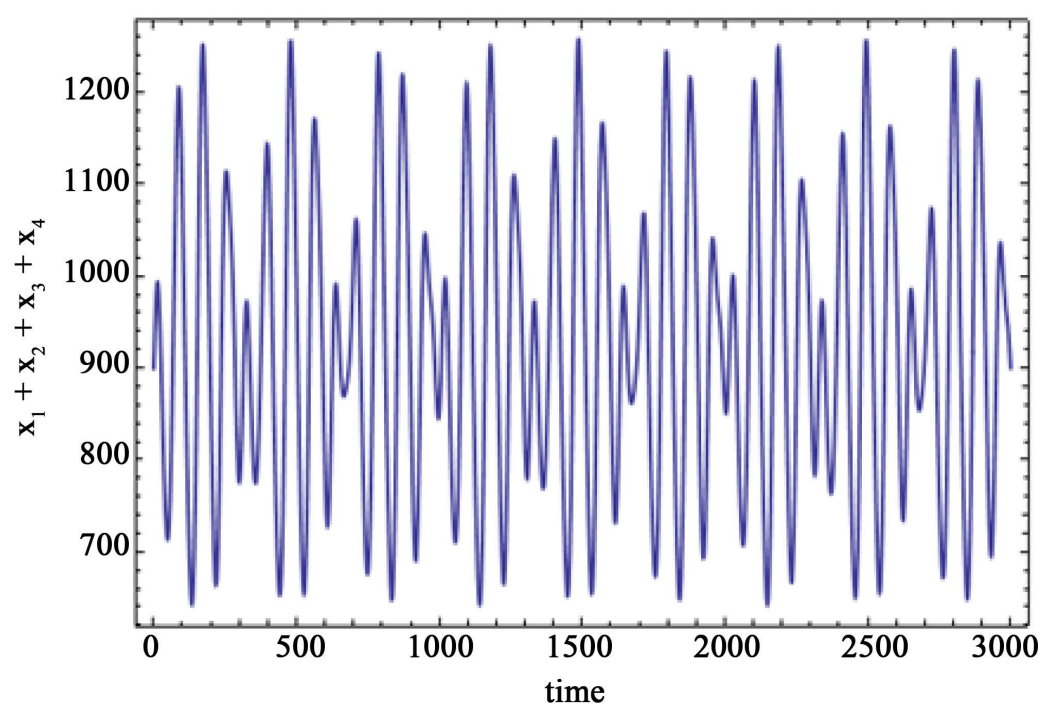

Figure 11. The sum of amplitude: $x_{1}+x_{2}+x_{3}+x_{4}$. The approximate periodicity $T \sim 1000$ can be observed from the data. 
Hence, conservative nonlinear interactions of component variables lead to a longer periodic time and a net stable system against external perturbations. In ecosystems, it could be interpreted so that living animals tend to have interactions with other animals as much as possible for their species to live long. The approximate periodic time of components and the net periodic time in nonlinear interactions may be one of important properties to understand stability of nonlinear interacting systems.

Our strategy of constructing solutions of nonlinear differential equations is to find a class of solutions having approximate periodic times. This may be true, and we investigated the class of solutions because the amplitude, $y$, and net periodic time, $T_{n}$, are important physical observables to understand nonlinear dynamical systems. Therefore, it could be understood that the stable periodicity, the standard rhythm, discussed in papers [7] [8] is the consequence of an approximate periodic phenomena of conservative nonlinear interactions.

The ten-year cycles of Canadian lynx and hare, interactions of microbes and other stable cycles are considered as examples of the approximate periodicity of nonlinear interacting systems. Hence, a possible condition to sustain stability of complex symbiotic systems can be examined by applying a conservative nonlinear differential equation.

The remarkable properties derived from conservative nonlinear interactions are summarized as follows:

1) The net periodic oscillation and time can be approximately defined in the conservative nonlinear interactions, and the net periodic time is longer than the periodic time of component variables: $T_{n}>T_{i}$

2) The 4-variable system becomes more stable than the 2-variable system against negative external perturbations. Nonlinear interactions of diverse component variables seem to maintain stability of the net system against negative external perturbations.

These results are different from those discussed in dissipative, nonconservative nonlinear interactions in many literatures which discuss limit cycles and attractors. The conservation law and durability of a conservative nonlinear system against external perturbations could be a key to understand stability of complex systems. The pattern simulations combined with external perturbations are possible for conserved nonlinear interactions, and it may be helpful to understand characteristic periodicities and responses of complex interacting systems.

\section{Conclusions}

In physics and engineering applications, it is standard to analyze physical quantities caused by heat conduction, electromagnetic and mechanical problems by way of the superposition principle [52] [53]. The superposition principle does not hold in nonlinear systems, and nonlinear interactions generate chaos in dynamical systems [54]. However, by applying the 2-variable solutions, we extracted periodic solutions to complex systems described with conservative nonlinear interactions. The superposition of component solutions exhibited an approximate net periodic amplitude and time, which is important to examine complex systems.

We reviewed characteristic properties for the 2-variable conservative nonlinear differential equation which generalizes Lotka-Volterra type prey-predator, competitive interactions, and explained that the atto-fox problem found in a simple Lotka-Volterra equation is not intrinsic to nonlinear differential equations. The 2-variable and 4-variable conservative nonlinear equations with external perturbations are useful to simulate real data numerically.

The conservative nonlinear interaction with external perturbations numerically suggested the existence of the standard rhythm (the 10-year population cycle) of Canadian lynx and snowshoe hare. The analysis of the 2variable conserved nonlinear equation indicates that nonlinear interactions could be a manifestation of strategy to live and thrive in nature [7] [8]. It is also confirmed in the 4-variable nonlinear model that the approximate periodic time of the net system is longer than each periodic time of component variables. A system in diverse interactions could be more stable than a simple system of 2-body interaction.

It is naturally observed that many complex systems from microscopic to macroscopic world seem to maintain stable structure, recoveries and self-organizations [48]-[51]. The conservation laws and symmetries could be a key to understand stability of complex systems. We employed the concept of conservation law or symmetry of Noether's theorem in dynamics. A conservative nonlinear interaction is defined with nonlinear interactions generated by a function $\Psi$ which may be considered as Hamiltonian or Lagrangian in dynamical systems. The $\Psi$-function can be used to check the accuracy of a numerical solution to the corresponding nonlinear differential equation [7] [8].

The solution of the 2-variable model is applied to construct solutions of the 4-variable conservative nonlinear 
equations. It is difficult to find an appropriate numerical solution for the 4-variable nonlinear differential equation, which tends to make numerical analyses hard to carry out. However, we showed a strategy to find a solution by employing the 2-variable solutions, which helps construct solutions and accomplish numerical analyses rigorously by employing $\Psi$-function. This approach can be extended to the 6 and 8 -variable conservative nonlinear equations.

The conservative nonlinear model has not been actively applied yet, though it revealed certain properties of the 10-year cycles of lynx and hare, microbe interactions. We are searching for ecological data or medical applications as microscopic interacting systems, such as diabetes or other chronic diseases exerted by multiple interacting factors. Collaborations to analyze big data with the current model are anticipated.

The nonlinear differential equation approach is useful to understand physical phenomena. We hope that the 4-variable conservative nonlinear equation may find useful and practical applications from microscopic complex systems to macroscopic ecosystems.

\section{References}

[1] Ramos, A.F., Innocentini, G.C.P., Forger, F.M. and Hornos, J.E.M. (2010) Symmetry in Biology: From Genetic Code to Stochastic Gene Regulation. IET Systems Biology, 4, 311-329. http://dx.doi.org/10.1049/iet-syb.2010.0058

[2] Ives, A.R. and Carpenter, S.R. (2007) Stability and Diversity of Ecosystems. Science, 317, 58-62.

[3] Allesina, S. and Tang, S. (2012) Stability Criteria for Complex Ecosystems. Nature, 483, 205-208. http://dx.doi.org/10.1038/nature10832

[4] Thébault, E. and Fontaine, C. (2010) Stability of Ecological Communities and the Architecture of Mutualistic and Trophic Networks. Science, 329, 853-856. http://dx.doi.org/10.1126/science.1188321

[5] Daafouz, J., Riedinger, P. and Iung, C. (2002) Stability Analysis and Control Synthesis for Switched Systems: A Switched Lyapunov Function Approach. IEEE Transactions on Automatic Control, 47, 1883-1887.

[6] Harberman, R. (1977) Mathematical Model: Population Dynamics, Prentice Hall, Upper Saddle River.

[7] Uechi, L. and Akutsu, T. (2012) Conservation Laws and Symmetries in Competitive Systems. Progress of Theoretical Physics Supplement, 194, 210-222. http://dx.doi.org/10.1143/PTPS.194.210

[8] Uechi, L. and Akutsu, T. (2013) Stability and Restoration Phenomena in Competitive Systems. Progress of Theoretical and Experimental Physics, 2013, $103 \mathrm{~J} 01$.

[9] Naz, R (2010) Conservation Laws for a Complexly Coupled KDV System, Coupled Burgers' System and Drinfeld-Sokolov-Wilson System via Multiplier Approach. Communications in Nonlinear Science and Numerical Simulation, 15, 1177-1182. http://dx.doi.org/10.1016/j.cnsns.2009.05.071

[10] Zhang, Y. and Zhou, Y. (2013) Symmetries and Conserved Quantities for Fractional Action-Like Pfaffian Variational Problems. Nonlinear Dynamics, 73, 783-793. http://dx.doi.org/10.1007/s11071-013-0831-x

[11] Basille, M., Fortin, D., Dussault, C., Ouellet, J.P. and Courtois, R. (2011) Ecologically Based Definition of Seasons Clarifies Predator-Prey Interactions. Ecography, 35, 1-10.

[12] Stenseth, N.C., Falck, W., Chan, K.S., Bjornstad, O.N., O’Donoghue, M., Tong, H., Boonstra, R., Boutin, S., Krebs, C., J. and Yoccoz, N.G. (1998) From Patterns to Processes: Phase and Density Dependencies in the Canadian Lynx Cycle. Proceedings of the National Academy of Sciences, 95, 15430-15435. http://dx.doi.org/10.1073/pnas.95.26.15430

[13] Schindler, D.E., Carter, J.L., Francis, T.B., Lisi, P.J., Askey, P.J. and Sebastian, D.C. (2012) Mysis in the Okanagan Lake Food Web: A Time-Series Analysis of Interaction Strengths in an Invaded Plankton Community. Aquatic Ecology, 46, 1-13. http://dx.doi.org/10.1007/s10452-012-9393-0

[14] Chyu, J., Fonarow, G.C., Tseng, C.H. and Horwich, T.B. (2014) Four-Variable Risk Model in Men and Women with Heart Failure. Circulation: Heart Failure, 7, 88-95. http://dx.doi.org/10.1161/CIRCHEARTFAILURE.113.000404

[15] Nair, S., Mishra, V., Hayden, K., Lisboa, P.J.G., Pandya, B., Vinjamuri, S., Hardy, K.J. and Wilding, J.P.H. (2011) The Four-Variable Modification of Diet in Renal Disease Formula Underestimates Glomerular Filtration Rate in Obese Type 2 Diabetic Individuals with Chronic Kidney Disease. Diabetologia, 54, 1304-1307. http://dx.doi.org/10.1007/s00125-011-2085-9

[16] Freedman, H.I. (1980) Deterministic Mathematical Models in Population Ecology. Marcel Dekker Inc, Basel.

[17] Lotka, A. (1925) Elements of Physical Biology. Williams and Wilkins, Baltimore.

[18] Volterra, V. (1926) Variazioni e fluttuazioni del numero d'individui in specie animali conviventi. Memoria della Reale Accademia Nazionale dei Lincei, 2, 31-113.

[19] Meinhardt, H. (1982) Models of Biological Pattern Formation. Vol. 6, Academic Press, London. 
[20] Gierer, A. and Meinhardt, H. (1972) A Theory of Biological Pattern Formation. Biological Cybernetics, 12, 30-39. http://dx.doi.org/10.1007/bf00289234

[21] Turing, A.M. (1952) The Chemical Basis of Morphogenesis. Philosophical Transactions of the Royal Society B, 237, 37-72. http://dx.doi.org/10.1098/rstb.1952.0012

[22] Kondo, S. and Asai, R. (1995) A Reaction-Diffusion Wave on the Skin of the Marine Angelfish Pomacanthus. Nature, 376, 765-768. http://dx.doi.org/10.1038/376765a0

[23] Lengyel, I. and Epstein, I.R. (1991) Modeling of Turing Structures in the Chlorite-Iodide-Malonic Acid-Starch Reaction System. Science, 251, 650-652. http://dx.doi.org/10.1126/science.251.4994.650

[24] Kumar, N. and Horsthemke, W. (2011) Effects of Cross Diffusion on Turing Bifurcations in Two-Species ReactionTransport Systems. Physical Review E, 83, Article ID: 036105. http://dx.doi.org/10.1103/physreve.83.036105

[25] Brauer, F. and Castillo-Chavez, C. (2010) Mathematical Models in Population Biology and Epidemiology. Springer Science, Berlin.

[26] Haccou, P., Jagers, P. and Vatutin, V.A. (2005) Branching Processes. Cambridge University Press, Cambridge. http://dx.doi.org/10.1017/CBO9780511629136

[27] Stenseth, N.C., Falck, W., Chan, K.S., Bjornstad, O.N., M. Donoghue, O., Tong, H., Boonstra, R., Boutin, S., Krebs, C.J. and Yoccoz. N.G. (1998) From Patterns to Processes: Phase and Density Dependencies in the Canadian Lynx Cycle. Proceedings of the National Academy of Sciences of the United States of America, 95, 15430-15435. http://dx.doi.org/10.1073/pnas.95.26.15430

[28] Elton, C. and Nicholson, M. (1942) The Ten-Year Cycle in Numbers of the Lynx in Canada. Journal of Animal Ecology, 11, 215-244. http://dx.doi.org/10.2307/1358

[29] Stenseth, N.C., Falck, W., Bjørnstad, O.N. and Krebs, C.J. (1997) Population Regulation in Snowshoe Hare and Canadian Lynx: Asymmetric Food Web Configurations between Hare and Lynx. Proceedings of the National Academy of Sciences of the United States of America, 94, 5147-5152. http://dx.doi.org/10.1073/pnas.94.10.5147

[30] Krebs, C.J., Boonstra, R., Boutin, S. and Sinclair, A.R.E. (2001) What Drives the 10-Year Cycle of Snowshoe Hares? BioScience, 51, 25-35. http://dx.doi.org/10.1641/0006-3568(2001)051[0025:WDTYCO]2.0.CO;2

[31] Basille, M., Fortin, D., Dussault, C., Ouellet, J.P. and Courtois, R. (2013) Ecologically Based Definition of Seasons Clarifies Predator-Prey Interactions. Ecography, 36, 220-229. http://dx.doi.org/10.1111/j.1600-0587.2011.07367.x

[32] Blasius, B., Huppert, A. and Stone, L. (1999) Complex Dynamics and Phase Synchronization in Spatially Extended Ecological Systems. Nature, 399, 354-359. http://dx.doi.org/10.1038/20676

[33] Maquet, J., Letellier, C. and Aguirre, L.A. (2007) Global Models from the Canadian Lynx Cycles as a Direct Evidence for Chaos in Real Ecosystems. Journal of Mathematical Biology, 55, 21-39. http://dx.doi.org/10.1007/s00285-007-0075-9

[34] Schindler, D.E., Carter, J.L., Francis, T.B., Lisi, P.J., Askey, P.J. and Sebastian, D.C. (2012) Mysis in the Okanagan Lake Food Web: A Time-Series Analysis of Interaction Strengths in an Invaded Plankton Community. Aquatic Ecology, 46, 1-13. http://dx.doi.org/10.1007/s10452-012-9393-0

[35] Gazi, N.H. (2012) Dynamics of a Marine Plankton System: Diffusive Instability and Pattern Formation. Applied Mathematics and Computation, 218, 8895-8905. http://dx.doi.org/10.1016/j.amc.2012.02.048

[36] Taniguchi, M., Bando, M. and Nakayama, A. (2008) Business Cycle and Conserved Quantity in Economics. Journal of the Physical Society of Japan, 77, Article ID: 114001. http://dx.doi.org/10.1143/jpsj.77.114001

[37] González, E. and Villena. M. (2011) Spatial Lanchester Models. European Journal of Operational Research, 210, 706715. http://dx.doi.org/10.1016/j.ejor.2010.11.009

[38] Mazenc, F. and Malisoff, M. (2010) Strict Lyapunov Function Constructions under LaSalle Conditions with an Application to Lotka-Volterra Systems. IEEE Transactions on Automatic Control, 55, 841-854. http://dx.doi.org/10.1109/TAC.2010.2041995

[39] Smith, H.L. and Waltman, P. (1995) The Theory of the Chemostat. Cambridge University Press, Cambridge. http://dx.doi.org/10.1017/CBO9780511530043

[40] Mollison, D. (1991) Dependence of Epidemic and Population Velocities on Basic Parameters. Mathematical Biosciences, 107, 255-287. http://dx.doi.org/10.1016/0025-5564(91)90009-8

[41] Kloeden, P.E. and Pötzsche, C. (2010) Dynamics of Modified Predator-Prey Models. International Journal of Bifurcation and Chaos, 20, 2657-2669. http://dx.doi.org/10.1142/S0218127410027271

[42] Goldstein, H., Poole, C. and Safko, J. (2001) Classical Mechanics. 3rd Edition, Addison Wesley, Upper Saddle River.

[43] Logan, J.D. (1977) Invariant Variational Principles. Academic Press Inc., Cambridge.

[44] Vujanovic, B.D. and Jones, S.E. (1989) Variational Methods in Nonconservative Phenomena. Academic Press Inc., 
Cambridge.

[45] Sieniutycz, S. (1994) Conservation Laws in Variational Thermo-Hydrodynamics. Kluwer Academic Pub., Norwell. http://dx.doi.org/10.1007/978-94-011-1084-6

[46] Sieniutycz, S. and Farkas, H. (2005) Variational and Extremum Principles in Macroscopic Systems. Elsevier, Amsterdam.

[47] Mattuck, R.D. (1976) A Guide to Feynman Diagrams in the Many-Body Problem. McGraw-Hill, New York.

[48] Prigogine, I. and Stengers, I. (1984) Order Out of Chaos. Bantam Books, New York.

[49] Nicolis, G. and Prigogine, I. (1977) Self-Organization in Nonequilibrium Systems. Wiley-Interscience Publication, Hoboken.

[50] Kauffman, S.A. (1993) The Origins of Order. Oxford University Press, New York.

[51] Bertalanffy, L.V. (1968) General System Theory. George Braziller Inc., New York.

[52] Jackson, J.D. (1962) Classical Electrodynamics. John Wiley \& Sons, Hoboken.

[53] Kreyszig, E. (1999) Advanced Engineering Mathematics. John Wiley \& Sons, Hoboken.

[54] Ott, E. (2002) Chaos in Dynamical Systems. Cambridge University Press, Cambridge. http://dx.doi.org/10.1017/CBO9780511803260 


\section{Appendix}

\section{Nonlinear Parameters in 4-Variable Lagrangian}

The numbering of nonlinear coefficients, $\alpha_{i}$ in the 4-variable Lagrangian in the Section 3, is shown in order to avoid complications. The nonlinear coefficients are numbered as:

$$
\begin{aligned}
\mathcal{L}= & \alpha_{1} \dot{x}_{1} x_{2}+\alpha_{2} x_{1} \dot{x}_{2}+\alpha_{3} \dot{x}_{3} x_{4}+\alpha_{4} x_{3} \dot{x}_{4}+\alpha_{5} x_{1} x_{1}+\alpha_{6} x_{1} x_{2}+\alpha_{7} x_{1} x_{3} \\
& +\alpha_{8} x_{1} x_{4}+\alpha_{9} x_{3} x_{1}+\alpha_{10} x_{3} x_{2}+\alpha_{11} x_{3} x_{3}+\alpha_{12} x_{3} x_{4}+\alpha_{13} x_{2} x_{1} \\
& +\alpha_{14} x_{2} x_{2}+\alpha_{15} x_{2} x_{3}+\alpha_{16} x_{2} x_{4}+\alpha_{17} x_{4} x_{1}+\alpha_{18} x_{4} x_{2}+\alpha_{19} x_{4} x_{3} \\
& +\alpha_{20} x_{4} x_{4}+\alpha_{21} x_{1} x_{2} x_{1}+\alpha_{22} x_{1} x_{2} x_{2}+\alpha_{23} x_{1} x_{2} x_{3}+\alpha_{24} x_{1} x_{2} x_{4} \\
& +\alpha_{25} x_{3} x_{4} x_{1}+\alpha_{26} x_{3} x_{4} x_{2}+\alpha_{27} x_{3} x_{4} x_{3}+\alpha_{28} x_{3} x_{4} x_{4}+c_{1} x_{2} \\
& +c_{2} x_{1}+c_{3} x_{4}+c_{4} x_{3} .
\end{aligned}
$$

and the nonlinear Equations (3.1)-(3.4), are derived from the lagrangian (5.2) by way of Euler-Lagrange equation:

$$
\begin{aligned}
\mathcal{L}= & \alpha_{1} \dot{x}_{1} x_{2}+\alpha_{2} x_{1} \dot{x}_{2}+\alpha_{3} \dot{x}_{3} x_{4}+\alpha_{4} x_{3} \dot{x}_{4}+\left(\alpha_{6}+\alpha_{13}\right) x_{1} x_{2} \\
& +\left(\alpha_{7}+\alpha_{9}\right) x_{1} x_{3}+\left(\alpha_{8}+\alpha_{17}\right) x_{1} x_{4}+\left(\alpha_{10}+\alpha_{15}\right) x_{2} x_{3} \\
& +\left(\alpha_{12}+\alpha_{19}\right) x_{3} x_{4}+\left(\alpha_{16}+\alpha_{18}\right) x_{2} x_{4}+\alpha_{5} x_{1}^{2}+\alpha_{11} x_{3}^{2} \\
& +\alpha_{14} x_{2}^{2}+\alpha_{20} x_{4}^{2}+\alpha_{21} x_{1}^{2} x_{2}+\alpha_{22} x_{1} x_{2}^{2}+\alpha_{27} x_{3}^{2} x_{4} \\
& +\alpha_{28} x_{3} x_{4}^{2}+\alpha_{23} x_{1} x_{2} x_{3}+\alpha_{24} x_{1} x_{2} x_{4}+\alpha_{25} x_{1} x_{3} x_{4} \\
& +\alpha_{26} x_{2} x_{3} x_{4}+c_{1} x_{2}+c_{2} x_{1}+c_{3} x_{4}+c_{4} x_{3} .
\end{aligned}
$$

The numbering of nonlinear coefficients of parentheses, $\left(\alpha_{6}+\alpha_{13}\right),\left(\alpha_{7}+\alpha_{9}\right), \cdots$, can be respectively handled as one independent parameter. The coefficients, $\alpha_{1}, \alpha_{2}, \alpha_{3}, \alpha_{4}$, are related to overall factors and become trivial in numerical calculations for (3.1)-(3.4). Hence, the number of nonlinear coefficients for the 4-variable conservative nonlinear equation is 22 . It should be noticed that if one directly starts from 4-variable nonlinear nonconservative differential equations in the same type, one is naturally directed to introduce 40 nonlinear coefficients.

The number of 4-variable nonlinear parameters is still large, but if 2-variable numerical solutions are used to construct the 4 -variable solution, one is supposed to control only 8 parameters. The method of solution is explained in the Section 3.1.

Submit or recommend next manuscript to OALib Journal and we will provide best service for you:

- Publication frequency: Monthly

- 9 subject areas of science, technology and medicine

- Fair and rigorous peer-review system

- Fast publication process

- Article promotion in various social networking sites (LinkedIn, Facebook, Twitter, etc.)

- Maximum dissemination of your research work

Submit Your Paper Online: $\underline{\text { Click Here to Submit }}$

Contact Us: service@oalib.com 